\title{
Multiple shocks near Mars
}

\author{
Eduard Dubinin $^{1,2,5}$, Konrad Sauer ${ }^{1,5}$, Klaus Baumgärtel ${ }^{3,5}$, and Krishna Srivastava ${ }^{4}$ \\ ${ }^{1}$ Max-Planck-Institut für Aeronomie, D-37191 Katlenburg-Lindau, Germany \\ ${ }^{2}$ Space Research Institute, Russian Academy of Sciences, 117810 Moscow, Russia \\ ${ }^{3}$ Astrophysikalisches Institut D-14437 Potsdam, Germany \\ ${ }^{4}$ University of Delhi, India \\ ${ }^{5}$ International Space Science Institute (ISSI), Hallerstrasse 6, 3012 Bern, Switzerland
}

(Received August 4, 1997; Accepted January 13, 1998)

\begin{abstract}
Mars presents us with an example of a magnetosheath in which the interaction of the solar wind with planetary plasma of heavy ions results in a generation of strong nonlinear bi-ion MHD waves. These waves provide oscillations in the momentum exchange between protons and heavy ions and may give rise to multiple shock-like structures. One-dimensional hybrid simulations of plasma flow interaction with a "heavy ion obstacle" were performed to study the generation of shock waves in bi-ion plasma. A differential motion of protons and heavy ions leads to bunching of heavy ion flow picked up by solar wind. Plasma bunching arises because of a resonant interaction between magnetosound waves excited by beam and "slow" waves of a spatial charge in the heavy ion flow. Both kinds of waves grow, steepen and gradually evolve to shocks.
\end{abstract}

\section{Introduction}

The problem of the formation of steepened magnetosound waves (shocklets) in mass-loaded solar wind has been widely discussed (Gary and Madland, 1988; Brinca and Tsurutani, 1989; Omidi and Winske, 1987, 1990; Omidi et al., 1994 and references therein). Omidi and Winske (1987) have distinguished two regimes of a mass-loading. For the quasiperpendicular case when the angle between flow velocity and the magnetic field $\alpha \geq 70^{\circ}$, a deceleration of the solar wind occurs through macroscopic electromagnetic fields and a shock arises from steepened fast magnetosound pulses. For oblique angles $60^{\circ} \geq \alpha \geq 20^{\circ}$, microscopic fields associated with the generation of low-frequency waves contribute essentially to the momentum exchange between solar wind protons and heavy ions. This case was analyzed by Omidi and Winske (1990) and Omidi et al. (1994). It has been suggested that an ion beam, formed by the ionization of cometary gas, generates magnetosound waves evolving to shocklets. Simulations performed by Omidi et al. (1994) have shown that, for a beam-ring distribution of heavy ions, the instability of parallel propagating nonresonant waves is saturated by the pitch-angle scattering of ions into halfshells, while oblique magnetosound modes continue to grow, leading to their dominance and evolution to shocks. These oblique steepened magnetosound waves give rise to shocks. For quasiperpendicular angles, Omidi and Winske $(1987,1990)$ have identified proton shocks without significant changes to the heavy ion fluid. In the present paper the additional discontinuity in heavies is found. It is worth noting that the model admits a differential streaming of ions, that is valid if the characteristic size of the system is less or comparable with the Larmor radius of heavies. The width of

Copy right $(C)$ The Society of Geomagnetism and Earth, Planetary and Space Science (SGEPSS); The Seismological Society of Japan; The Volcanological Society of Japan; The Geodetic Society of Japan; The Japanese Society for Planetary Sciences. the Martian magnetosheath is much less than the pick-up $\mathrm{O}^{+}$ gyroradius. In such systems, the widely used approach of the pick-up ring-like distribution of heavies which evolves to a shell distribution as a result of the pitch-angle diffusion on Alfvén waves (see, for example, Galeev et al., 1991) is not appropriate. The treatment used in this paper is based on biion MHD equations for Large Larmor Systems. The relative streaming of ion species may lead to a resonant interaction between "heavy ion and proton magnetosound" waves and a subsequent bunching of the heavy ion flow. We attribute the generation of multiple shock-like structures in the Martian magnetosheath and in the 1-D hybrid simulations to a coupling between both ion species with a differential ion streaming.

\section{Observations}

The lack of a strong magnetic screen at Mars which is able to stop the solar wind leads to the direct interaction of two plasmas, whereas a massive planetary plasma acts as an "obstacle" for the solar wind. Measurements made by Phobos spacecraft near Mars found that a transition from shocked solar wind dominated by protons to planetary plasma often occurs by steps via a set of shock-like structures. Figure 1 shows an example of variations of plasma parameters in the magnetosheath. Step-like deceleration of the solar wind is observed. An obvious periodical heating of protons which replicates subsequent drops of bulk velocity and followed by a plasma "cooling', emphasizes an analogy with multiple shocks. Bunches of heavy ions and protons are clearly seen in the variations of the ratio of oxygen and proton number densities (Fig. 2). Stratification of plasma on alternating layers of protons/heavies is revealed from the comparison of the behavior of proton and electron number densities (Dubinin et al., 1996). When $n_{\mathrm{p}} \rightarrow n_{\mathrm{e}}$, plasma is occupied mostly by protons. A divergence of curves of $n_{\mathrm{e}}$ and $n_{\mathrm{p}}$ indicates the 
PHOBOS-2

FEBRUARY 15, 1989

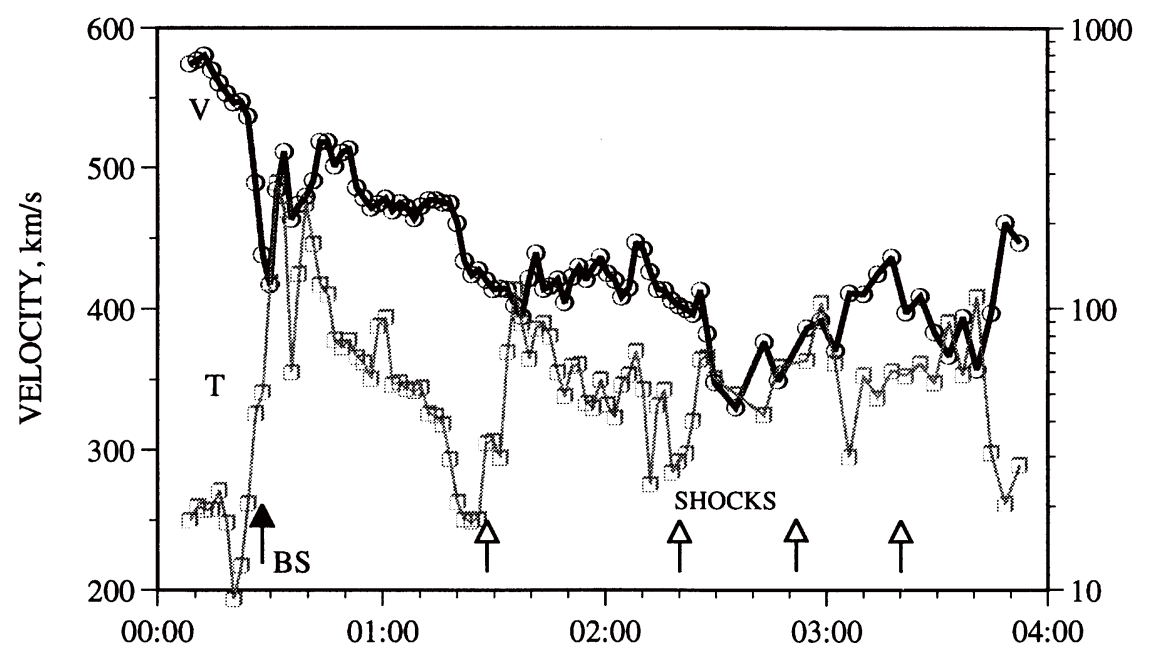

告

Fig. 1. Bulk proton velocity and temperature of protons for the pass through the magnetosheath of Mars on 15 February 1989. Step-like deceleration of the protons accompanied by their heating indicates the presence of multiple shocks.

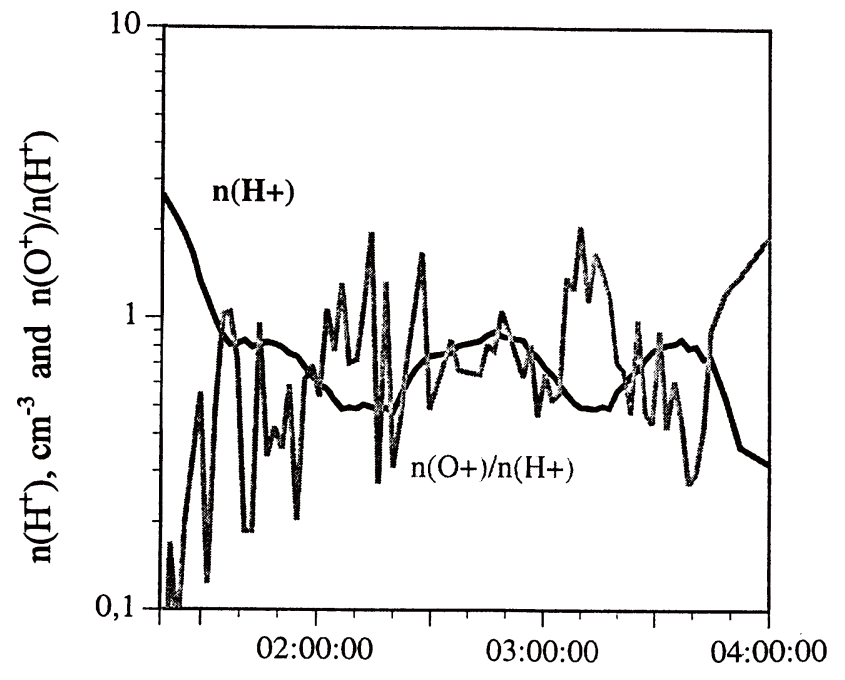

Fig. 2. Number density of protons and the ratio of oxygen and proton number densities.

transition from proton plasma to a layer filled by the planetary plasma. Dubinin et al. (1996) have given a qualitative scheme illustrating a formation of these structures (Fig. 3). Near clumps of heavies, an electric field, associated with plasma and magnetic field gradients and with differential motions of ion species $\left(e E n_{\mathrm{e}}=-\left(\nabla P_{\mathrm{e}}+\nabla B^{2} / 8 \pi-\boldsymbol{B} \nabla \boldsymbol{B} / 4 \pi\right.\right.$ $\left.+e / c \sum n_{\mathrm{i}} \boldsymbol{V}_{\mathrm{i}} \times \boldsymbol{B}\right)$, decelerates incoming protons. If the height of electrostatic potential is high enough, a significant fraction of protons can be reflected. Transmitted protons are accelerated at the rear side of electrostatic potential giving rise to an observed increase of proton velocity. A proton heating is provided by the compression of proton fluid, as well as by the contribution of reflected protons which gain energy in the motional electric field and pass through the shock with a high thermal spread of velocity. A subsequent set of such structures may result in a step-like transition via multiple shocks to the plasma dominated by planetary ions. Hybrid simulations of the solar wind interaction with a

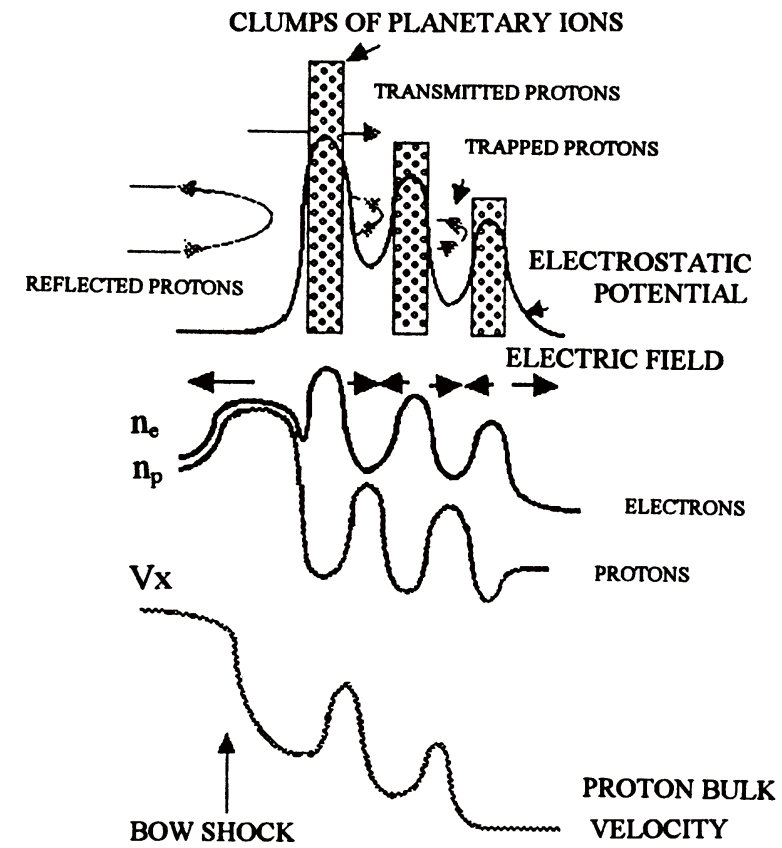

Fig. 3. A scheme of multiple shock transition taken from observations.

cometary plasma made by Omidi and Winske (1990) have demonstrated the appearance of multiple shocklets and a step-like transition to subsonic solar wind. The purpose of this paper is to address a question about the generation of multiple shock-like structures near Mars.

\section{1-D Hybrid Simulations}

One-dimensional hybrid simulations of the interaction of plasma flow with a cloud of heavy ion plasma were performed. Ions of each species were described as particles and electrons as a mass-less fluid. The spatial values were scaled to the proton inertial length $L\left(L=\mathrm{v}_{\mathrm{A}} / \omega_{\mathrm{Bp}}\right.$, where $\omega_{\mathrm{Bp}}=e B /$ $m_{\mathrm{p}} c$, and $\left.\mathrm{v}_{\mathrm{A}}=B /\left(4 \pi n_{\mathrm{p}} m_{\mathrm{p}}\right)^{1 / 2}\right)$, and the time quantities were scaled to the inverse proton gyrofrequency $T=\omega_{\mathrm{Bp}}{ }^{-1}$. A grid 
with spacings of $0.5 \mathrm{~L}$ and time steps of $0.001-0.02 T$ were used. The mean number of macroparticles in a cell was about $25 \div 40$ per ion species. The mass ratio of heavy ions was taken to be between 12 and 20. Plasma parameters were normalized to proton upstream values. Protons with predetermined parameters were injected into the box from the left side. A gaseous cloud of neutral atoms with $m_{\mathrm{h}}=12 \div 20$, which was located at $X=0$, forms an obstacle by a production of ionized population. The obstacle also generates a biion flow by adding newly ionized heavy ions to the solar wind. The localized at $X=0$ obstacle of a weak ion production with a Gaussian profile was immersed into the incoming solar wind flow $\left(M_{\mathrm{A}}=5\right)$. The magnetic field was nearly perpendicular to the flow velocity $\left(\alpha=85^{\circ}\right)$ and perpendicular to the $X Y$ plane. A normalized gas production was $Q_{\mathrm{h}}$ $=0.0375\left(Q_{\mathrm{h}}=n_{\mathrm{o}} v_{\mathrm{ph}} / n_{\mathrm{po}} \omega_{\mathrm{Bp}}\right.$, where $n_{\mathrm{o}} v_{\mathrm{ph}}$ is the ion production rate in the center of the cloud, $n_{\mathrm{o}}$ is the maximum neutral gas density, $v_{\mathrm{ph}}$ is the photoionization rate, and $n_{\mathrm{po}}$ is the undisturbed proton density).

Simulations demonstrate a coupling between ion species. Figure 4 shows variations in the fluid parameters of protons and heavy ions along the $\mathrm{X}$-axis at different times, $T=100$, $200,300,400$. Newly ionized particles, originating near the source, begin to move on cycloid trajectories, taking the momentum from incoming solar wind protons. The sequence of processes, which are responsible for the strong electromagnetic coupling between protons and heavies, has been discussed by Omidi and Winske (1987). When heavy ions are near the rest, the electron fluid slows down to provide a charge neutrality $\mathrm{v}_{\mathrm{ex}} \sim n_{\mathrm{p}} \mathrm{v}_{\mathrm{p}} /\left(n_{\mathrm{p}}+n_{\mathrm{h}}\right)$. Deceleration of electrons leads to a decrease in the motional electric field $E_{y}=$ $-1 / c\left(\mathrm{v}_{\mathrm{ex}} B_{z}\right)$ which causes the proton flow to be deflected in the $-Y$ direction which is opposite to the direction of the motional electric field $E_{y}=-1 / c\left(\mathrm{v}_{x} B_{z}\right)>0$ :
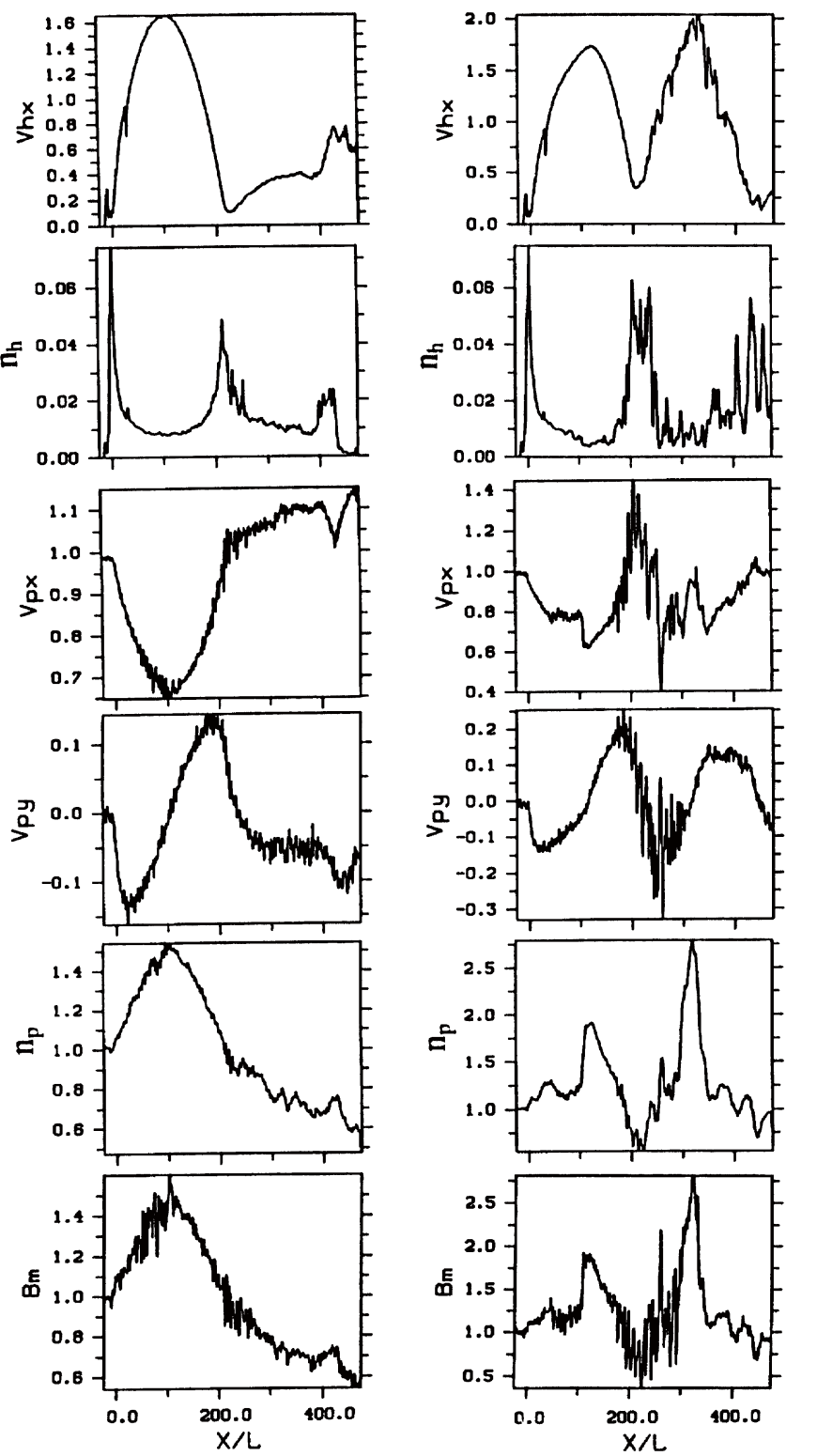
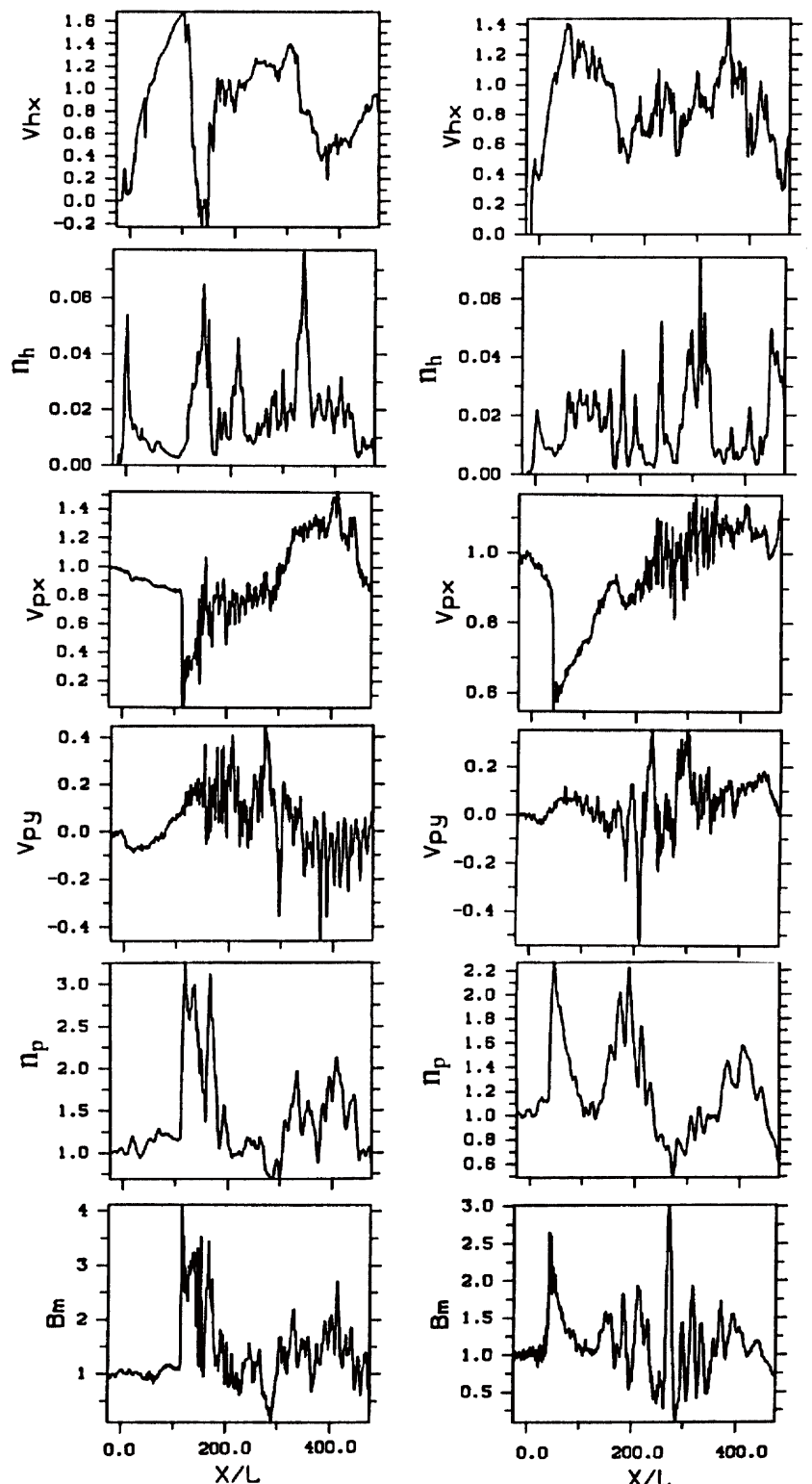

Fig. 4. Variations of plasma parameters along the $X$-axis at different times in 1-D hybrid simulations of plasma flow past a localized heavy ion source. Plasma parameters are normalized to the proton upstream values. Distance and time are normalized to $\mathrm{v}_{\mathrm{Ap}} / \omega_{\mathrm{Bi}}$ and $\omega_{\mathrm{Bi}}{ }^{-1}$. 


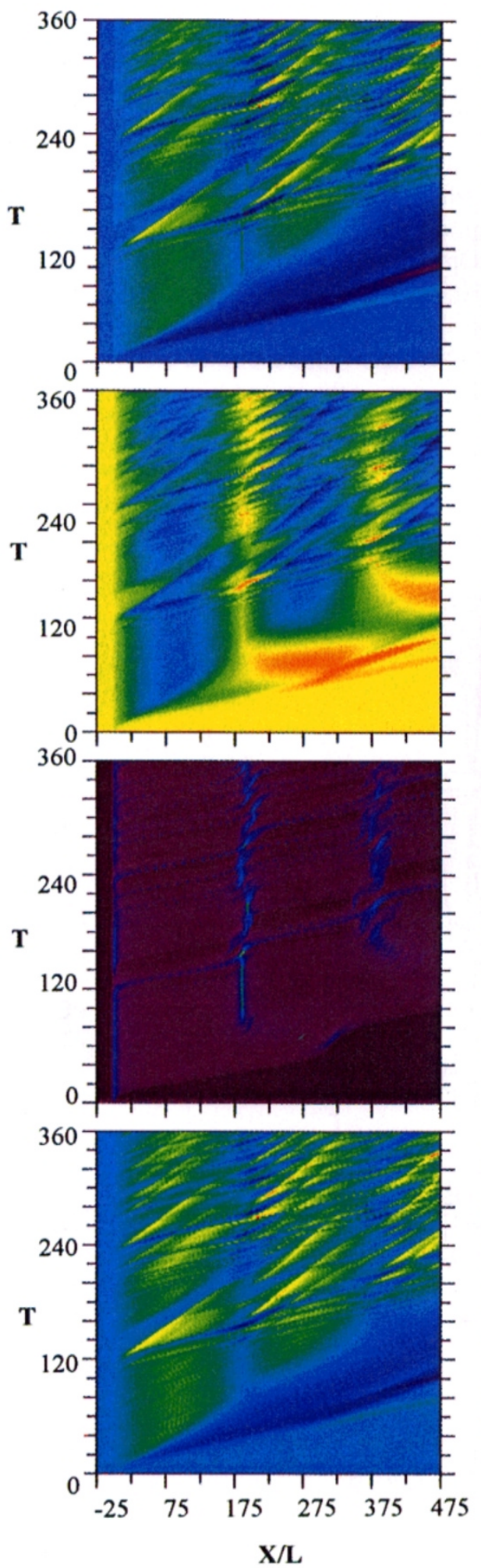

a)
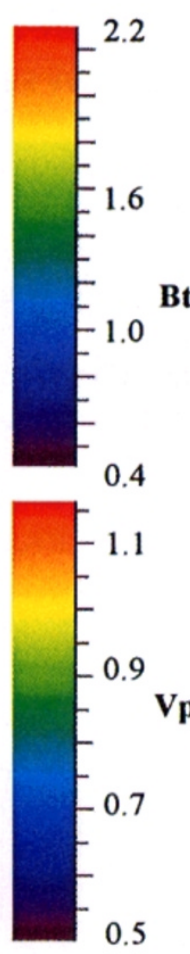

0.5
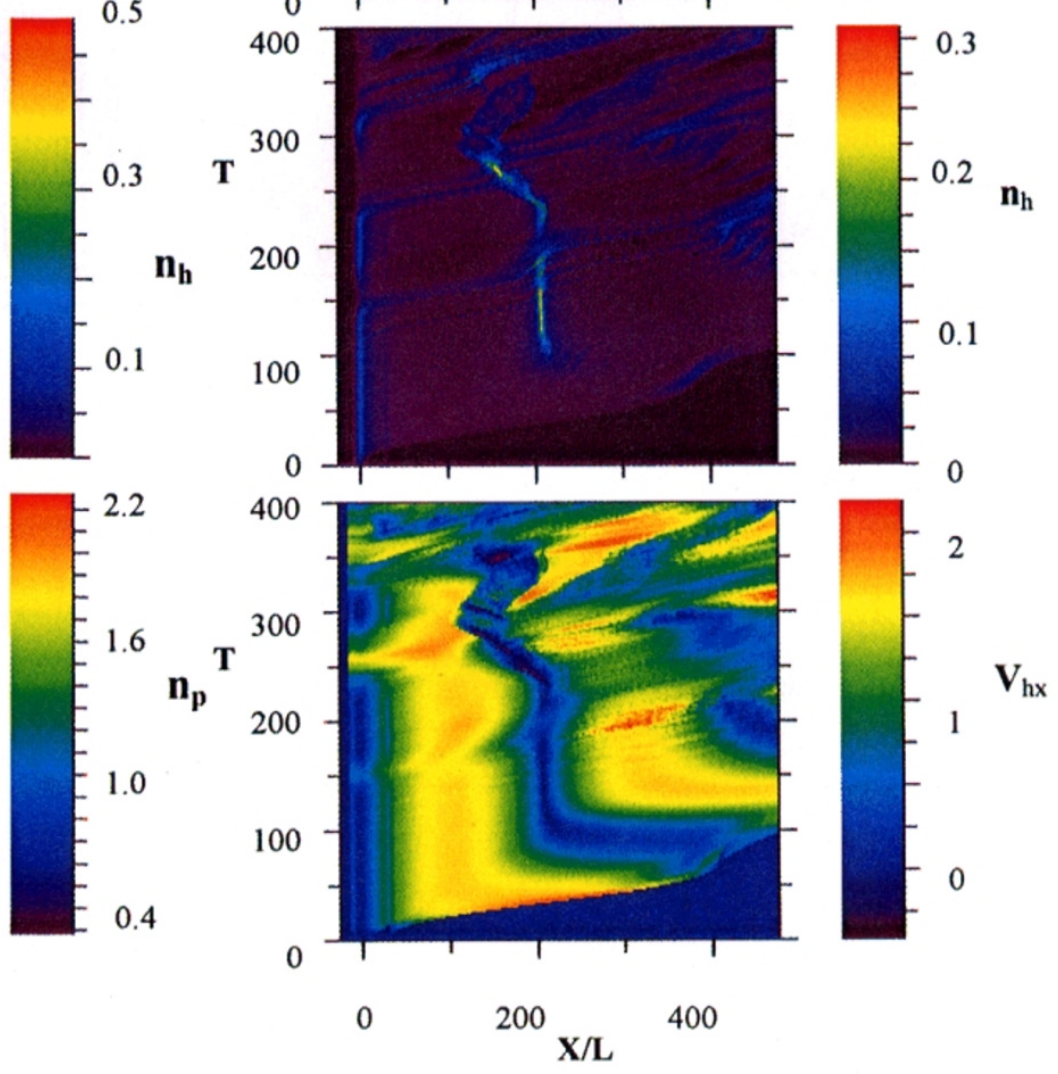

b)

Fig. 5. a) Spacetime diagram of the magnetic field value, the $x$-component of the velocity of heavies, and number densities of heavies and protons. The meaning of the color plots is clear from the color bars of the corresponding parameters. A bunching of plasma flow and the appearance of nonlinear waves propagating downstream is observed. b) Spacetime diagram of the $B_{z}, n_{\mathrm{p}}, n_{\mathrm{h}}$, and $\mathrm{v}_{\mathrm{h} x}$, which illustrates the case when shocks propagating in the upstream direction arise. Snapshots of plasma variations for this run are given in Fig. 4. 


$$
\frac{d \mathrm{v}_{p y}}{d t}=\frac{e}{m_{p}}\left(E_{y}-\frac{\mathrm{v}_{p x} B_{z}}{c}\right)<0 .
$$

The deflection of protons is clearly observed in the fourth panel from the top (the first column of Fig. 4). The arising Lorentz force $1 / c\left(\mathrm{v}_{y} B_{z}\right)$ decelerates the streaming protons which transfer the momentum to heavy ions. The bulk speed of heavies gradually exceeds the proton velocity. To supply a charge quasi-neutrality electrons begin to outrun protons that provides an enhancement of the electron bulk velocity $\mathrm{v}_{\mathrm{ex}}$ and an increase in the motional electric field $E_{y}$. Proton flow is deflected in the opposite, $+Y$ direction and the Lorentz force $1 / c\left(\mathrm{v}_{y} B_{z}\right)$ accelerates the protons to suppress their lag behind the heavies. As a result, the heavies transfer the momentum back to the protons. A motion of both ion populations begins gyrating and the exchange of momentum between ion flows occurs in a periodical manner. The process of alternating acceleration/deceleration of heavies/ protons becomes more clear if we write the momentum equations for protons and heavies in bi-ion plasma with differential streaming (Sauer et al., 1998):

$m_{\mathrm{p}} \frac{d \mathrm{v}_{\mathrm{p}}}{d t}=\frac{e n_{\mathrm{h}}}{c n_{\mathrm{e}}}\left(\mathrm{v}_{\mathrm{p}}-\mathrm{v}_{\mathrm{h}}\right) \times B-\frac{\nabla P_{\mathrm{e}}}{n_{\mathrm{e}}}-\frac{\nabla P_{\mathrm{p}}}{n_{\mathrm{p}}}-\frac{\nabla B^{2}}{8 \pi n_{\mathrm{e}}}+\frac{B \cdot \nabla B}{4 \pi n_{\mathrm{e}}}$,

$m_{\mathrm{h}} \frac{d \mathrm{v}_{\mathrm{h}}}{d t}=\frac{e n_{\mathrm{p}}}{c n_{\mathrm{e}}}\left(\mathrm{v}_{\mathrm{h}}-\mathrm{v}_{\mathrm{p}}\right) \times B-\frac{\nabla P_{\mathrm{e}}}{n_{\mathrm{e}}}-\frac{\nabla P_{\mathrm{h}}}{n_{\mathrm{h}}}-\frac{\nabla B^{2}}{8 \pi n_{\mathrm{e}}}+\frac{B \cdot \nabla B}{4 \pi n_{\mathrm{e}}}$.

Indeed, the Lorentz force, related with a differential streaming of ion fluids $(\approx \Delta \mathbf{v} \times \boldsymbol{B})$, has the opposite action on protons and heavies. A "larmoring" of ion populations occurs because either ion fluid in their reference frame senses the motional electric field $-(1 / c) \Delta \mathbf{v} \times \boldsymbol{B}$ which is supplied by differential ion streaming.

At later times, a differential motion of the ions becomes unstable and a bunching on a smaller scale occurs $(T=200)$. Small-scale structuring of $n_{\mathrm{h}}$ gives rise to subsequent variations of the plasma parameters in the same manner as has been discussed above. Bi-ion compression waves, which are generated in the process of bunching, gradually steepen and give rise to shocklets propagating in the upstream $(-X)$ direction. At $T=300$ and $T=400$ these shock-like structures are visible. After the passage of these shocks, the bulk velocities of protons and heavy ions are approximately equal although strong smaller-scale fluctuations remain.

Bunching and oscillations in bi-ion plasma are clearly recognized in the plots of Fig. 5 which presents a space-time diagram of plasma/field variations. Figure 5a depicts the results of simulations for the case when shocks were not yet generated. Nonlinear waves, carried by the plasma flow downstream, appear at $\sim 130 T$. Figure 5 b shows that large amplitude waves gradually steepen and give rise to shocks. A formation of shocks is identified by an abrupt change of the propagating direction. Two shocks moving in the upstream direction at different speeds may be found in Fig. 5 b. A "proton shock" is seen in the panels of $n_{\mathrm{p}}$ or $\mathrm{v}_{\mathrm{p}}$. Another shock, which is seen in the $n_{\mathrm{h}}$ panel, propagates with a
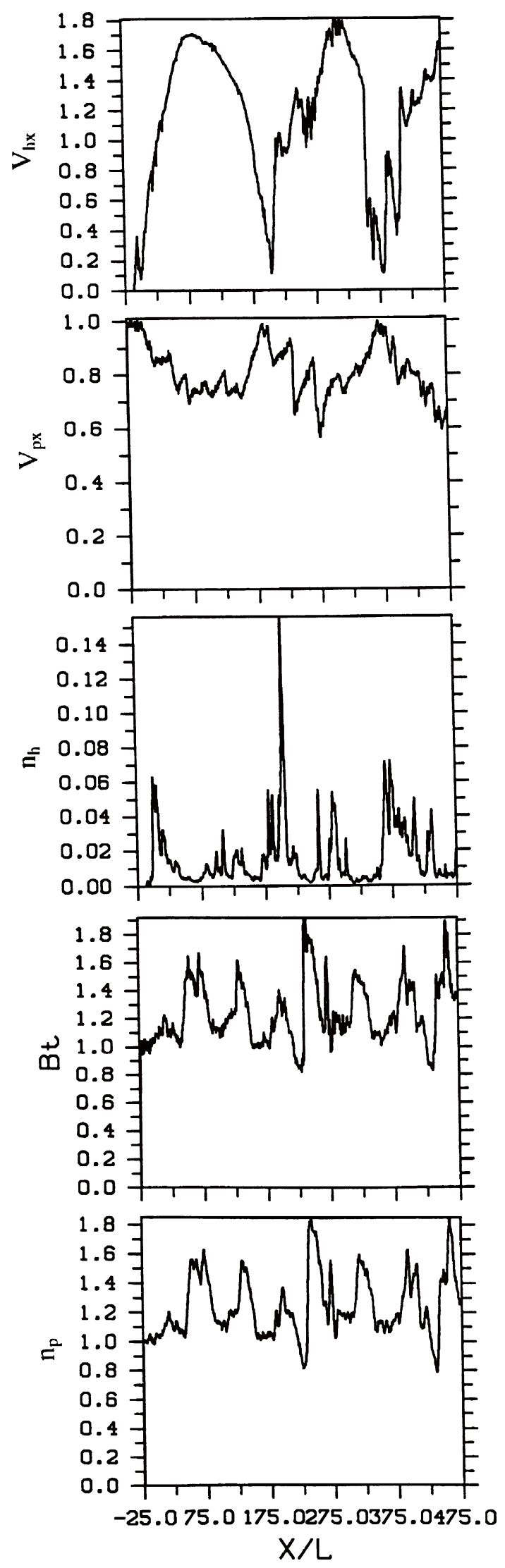

Fig. 6. Example of nonlinear steepened waves arising due to the bunching of plasma flow. The double peak distribution of $n_{\mathrm{p}}$ and $B_{t}$ is the effect of two waves propagating with different velocities. 
slower speed. The effects of collisions of shocks with nonlinear waves traveling downstream are also revealed. Shock-like structures are clearly observed in Fig. 6 which gives a snapshot of the plasma distribution at $T=300$.

A periodicity in the process of momentum exchange between protons and heavies is clearly observed before the formation of multiple shocks (Fig. 5). The driving force of these oscillations in the momentum exchange is the Lorentz force related with a differential streaming of ion flows $(\sim(1 /$ c) $\Delta \mathbf{v} \times \boldsymbol{B})$, and the forces arising due to the gradients of the magnetic field and plasma pressures, which either acceler-
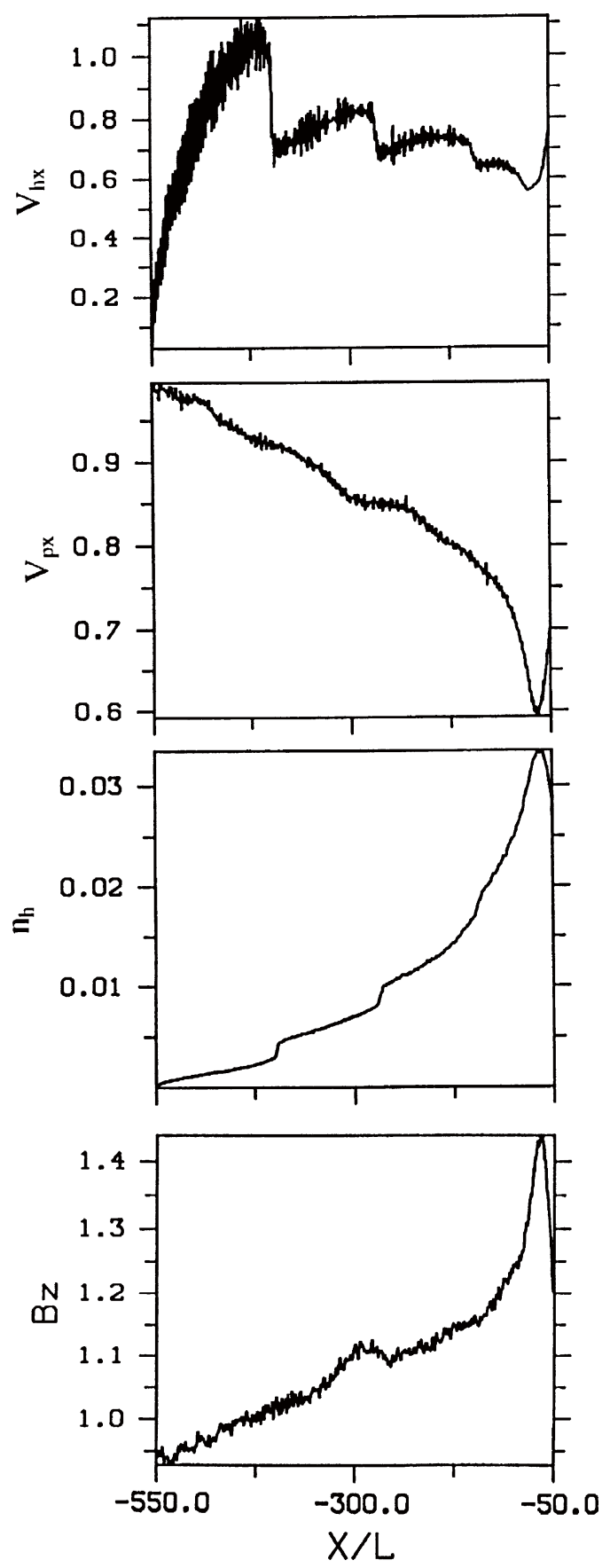

Fig. 7. Distribution of the speeds of heavies $\left(\mathrm{v}_{\mathrm{h} x}\right)$, and protons $\left(\mathrm{v}_{\mathrm{p} x}\right)$, the heavy ion density and the $z$-component of the magnetic field along the $X$-axis for $T=300$ in 1D-hybrid simulations of solar wind interaction with an extended obstacle $\left(M_{\mathrm{A}}=2.5\right)$. A step-like momentum transfer between protons and heavies is clearly seen. ate/decelerate heavies/protons or versus decelerate/accelerate heavies/protons. When waves steepen and multiple shocks are formed, the momentum transfer is realized in steps. A step-like deceleration of protons is distinctly revealed in 1-D hybrid simulations. Figure 7 shows the spatial distribution of the plasma parameters for 1-D hybrid simulations with an extended obstacle. Heavy ions start to flow along a cycloid trajectory and achieve upstream proton velocity. Then, an abrupt deceleration of the heavies happens. The heavies undergo a sharp transition and their bulk speed decreases to a value that is less than the proton speed. The subsequent reacceleration of the heavy ions by the $\Delta \mathbf{v} \times \boldsymbol{B}$ force is followed by the next deceleration and the process repeats but an increment of heavy ion velocity becomes smaller, because of an increase in the total mass of heavies taking momentum from the proton flow. Variations of the proton bulk velocity occur in an anti-phase with the velocity of the heavies.

\section{Discussion}

A prominent feature of 1-D hybrid simulations is the distinct bunching of the heavy ion flow. It is suggested that bunching is caused by an ion beam-plasma interaction. Waves of the spatial charge of a beam in plasma are described by the dispersion relation $\pm \omega_{\text {beam }}=\omega-k \Delta \mathrm{v}$. The signs \pm correspond to so called fast and slow waves. For an ion beam in unmagnetized plasma, these waves may be considered as ion sound waves propagating in both directions. If a slow mode $\left(\Delta \mathrm{v}-\omega_{\text {beam }} / k\right)$, which is a wave of negative energy, is synchronized with a plasma wave $\omega / k$ of positive energy, generated by a beam, then instability develops because bunches of the density of a beam come to the resonance with bunches in plasma and grow with time: $\Delta \mathrm{v}-\omega_{\text {beam }} / k \approx \omega / k$.

Addressing the question of the origin of waves, consider Eqs. (1)-(5) given in a related paper by Sauer et al. (1997). Using these equations together with the condition of quasineutrality $n_{\mathrm{e}}=n_{\mathrm{p}}+n_{\mathrm{h}}$ and making the assumptions about isothermal electrons $P_{\mathrm{e}}=n_{\mathrm{e}} k T_{\mathrm{e}}$ and cold ions $T_{\mathrm{p}}, T_{\mathrm{h}}<<T_{\mathrm{e}}$, we can easily obtain the dispersion relation for the case of $\boldsymbol{B}$ pointing out the simulation plane. If we neglect, for simplicity, the first terms in (2)-(4) of Sauer et al. (1997), the dispersion relation has a similar form as that for unmagnetized plasma (McKenzie et al., 1993):

$$
\left[\left(\mathrm{v}_{\mathrm{ph}}-\mathrm{v}_{\mathrm{p}}\right)^{2}-c_{\mathrm{Mpo}}^{2}\right] \cdot\left[\left(\mathrm{v}_{\mathrm{ph}}-\mathrm{v}_{\mathrm{h}}\right)^{2}-c_{\mathrm{Mho}}^{2}\right]=c_{\mathrm{Mpo}}^{2} c_{\mathrm{Mho}}^{2}
$$

where $v_{\mathrm{p}}$ and $\mathrm{v}_{\mathrm{h}}$ are bulk speeds of protons and heavies, $\mathrm{v}_{\mathrm{ph}}$ is the phase velocity, $\mathrm{v}_{\mathrm{ph}}=\omega / k$, and

$$
\begin{array}{ll}
c_{\mathrm{Mp}}^{2}=\mathrm{v}_{\mathrm{Ap}}^{2} \frac{n_{\mathrm{p}}^{2}}{n_{\mathrm{e}}^{2}}+c_{\mathrm{sp}}^{2} \frac{n_{\mathrm{p}}}{n_{\mathrm{e}}}, & c_{\mathrm{Mh}}^{2}=\mathrm{v}_{\mathrm{Ah}}^{2} \frac{n_{\mathrm{h}}^{2}}{n_{\mathrm{e}}^{2}}+c_{\mathrm{sh}}^{2} \frac{n_{\mathrm{h}}}{n_{\mathrm{e}}}, \\
\mathrm{v}_{\mathrm{Ap}}=\frac{B}{\left(4 \pi n_{\mathrm{p}} m_{\mathrm{p}}\right)^{1 / 2}}, & \mathrm{v}_{\mathrm{Ah}}=\frac{B}{\left(4 \pi n_{\mathrm{h}} m_{\mathrm{h}}\right)^{1 / 2}}, \\
c_{\mathrm{sp}}=\left(\frac{T_{\mathrm{e}}}{m_{\mathrm{p}}}\right)^{1 / 2}, & c_{\mathrm{sh}}=\left(\frac{T_{\mathrm{e}}}{m_{\mathrm{h}}}\right)^{1 / 2}
\end{array}
$$


are magnetosound, Alfvén and sound speeds for protons and heavies in bi-ion plasma. Within the proton reference frame, the dispersion relation may be written in the form:

$$
\left[\mathrm{v}_{\mathrm{ph}}^{2}-c_{\mathrm{Mp}}^{2}\right] \cdot\left[\left(\mathrm{v}_{\mathrm{ph}}-\Delta \mathrm{v}\right)^{2}-c_{\mathrm{Mh}}^{2}\right]=c_{\mathrm{Mp}}^{2} c_{\mathrm{Mh}}^{2},
$$

where $\Delta \mathrm{v}$ is the differential velocity of heavy ions and protons, $\Delta \mathrm{v}=\mathrm{v}_{\mathrm{h}}-\mathrm{v}_{\mathrm{p}}$. If the relative streaming and abundance of heavies is small, i.e., $\Delta \mathrm{v} \sim 0$ and $n_{\mathrm{h}} / n_{\mathrm{p}}<<1$, the existence of two modes follows from the dispersion relation:

$$
\begin{aligned}
& \mathrm{v}_{\mathrm{ph}}^{(1)} \approx c_{\mathrm{Mp}}, \\
& \mathrm{v}_{\mathrm{ph}}^{(2)} \approx c_{\mathrm{Mh}} .
\end{aligned}
$$

These modes may be referred to as "proton" and "heavy ion" modes. A differential streaming of ion populations may lead to a coupling between these modes. If abundance of heavies is much less than that of protons $\left(c_{\mathrm{Mh}}<<c_{\mathrm{Mp}}\right)$, the velocity of a slow mode, traveling with the beam, is mainly determined by the convection velocity of that beam $(\Delta \mathrm{v})$. A beam of heavies generates magnetosound waves $\left(c_{\mathrm{Mp}}\right)$ in "proton plasma". Bunches of the plasma density in this wave will grow if they come to the resonance with bunches in the heavy ion flow, and for plasma oscillations propagating in the $\varphi$-direction, resonance between waves may happen $\left(c_{\mathrm{Mp}}\right.$ $\left.\approx \Delta \mathrm{v} \cos \varphi-c_{\mathrm{Mh}}\right)$. The scheme in Fig. 8 illustrates the geometry of the resonance conditions. At any point along the cycloid trajectory of heavies the relative velocity of heavies and protons is $\Delta \mathrm{v}$. Slow mode, carried by heavy ion beam, can resonantly interact with magnetosound waves $\left(\mathrm{v}_{\mathrm{ph}} \sim c_{\mathrm{Mp}}\right)$ generated in the proton plasma. Two-dimensional bi-ion MHD simulations confirm this pattern. Multiple Mach cones emanate from knots of bunched flow of heavy ions (Fig. 9). In their turn, the knots track the direction of the slow mode carried by the ion beam.

It is worth noting that two modes, traveling in the proton flow, may be found from the panel of $n_{\mathrm{p}}$ (Fig. 5a). The velocities of these modes in the laboratory reference frame are about of $5.3 \mathrm{v}_{\mathrm{Ap}}$ and $1.8 \mathrm{v}_{\mathrm{Ap}}$. Perturbations in heavy ion density are carried by ion flow with higher velocity $6.8 v_{A p}$. One interesting feature is that values of these velocities don't vary although the relative speed of the heavy ions and protons varies essentially. A plausible explanation is that slow wave, carried by heavies, travels in the $\Delta v$-direction in the proton reference frame (Fig. 8). In the laboratory system, the wave propagates along a "cycloid trajectory" $\left(\Delta \mathrm{v}+\mathrm{v}_{\mathrm{p}}=\right.$ $\left.\mathrm{v}_{\mathrm{h}}\right)$ of heavies. Therefore, $\left(\omega / k_{x}=\mathrm{v}_{\mathrm{h}} / \cos \theta\right)$, where $\theta$ is the angle between $\mathrm{v}_{\mathrm{h}}$ and the $X$-axis. The $\theta$-angle for a cycloid trajectory is $\cos \theta=\mathrm{v}_{\mathrm{h}} / 2 a \omega_{\mathrm{Bh}}$. Then, $\omega / k_{x}=2 a \omega_{\mathrm{Bh}}$, where $2 a$ is the height of the cycloid $\left(a=\mathrm{v}_{\mathrm{p}} / \omega_{\mathrm{Bh}}\right)$, and does not depend on the local speed of the heavy ion flow. In reality, both ion fluids, heavies and protons, move along modified cycloid gyrating around each other with a bi-ion cut-off frequency $\Omega^{*}=\omega_{\mathrm{Bp}}\left(n_{\mathrm{h}} / n_{\mathrm{e}}\right)+\omega_{\mathrm{Bh}}\left(n_{\mathrm{p}} / n_{\mathrm{e}}\right)$. The height of a modified cycloid, which describes the motion of heavies, is smaller due to momentum exchange between ion species. The wavelength $S_{\text {mod }}$ of the modified cycloid is about of $250 \mathrm{~L}$, instead of $370 L$ for an ideal cycloid which describes the
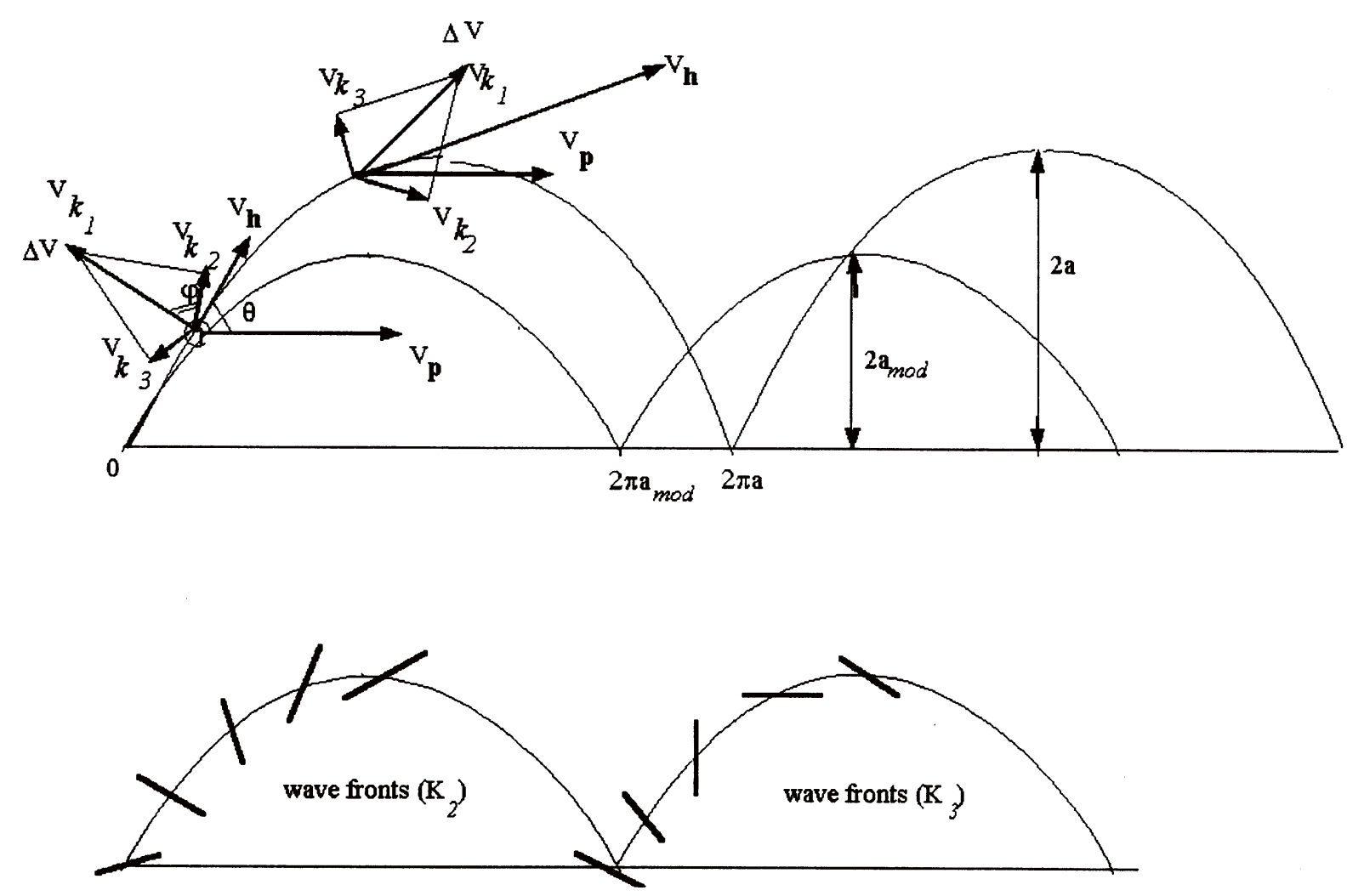

Fig. 8. Sketch illustrating the generation of a wave pattern due to bi-ion coupling between proton and heavy ion flows. 


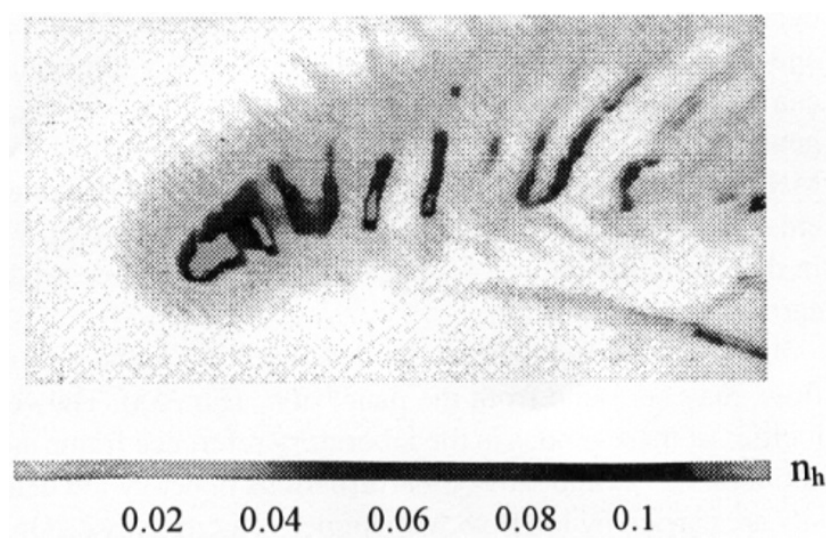

Fig. 9. A bi-ion 2-D pattern of solar wind flow past a local heavy ion source (reproduced from Bogdanov et al., 1996) $\left(M_{\mathrm{A}}=10\right)$. Bunching of a heavy ion beam traveling along a cycloid-type trajectory is seen. Two waves emanating from knots of the gyromotion of the heavies and the slow mode carried by the heavy ion beam correspond to waves which resonantly interact due to the differential motion of ion fluids.

motion of a test particle $\left(S_{\mathrm{id}}=2 \pi a=2 \pi\left(\mathrm{v}_{\mathrm{po}} / \omega_{\mathrm{Bh}}\right)\right.$, where $\mathrm{v}_{\mathrm{po}}$ $\left.=5 \mathrm{v}_{\mathrm{Ap}}, \omega_{\mathrm{Bh}}=\omega_{\mathrm{Bp}} / 12\right)$. Therefore, the phase velocity of the slow mode in the laboratory system may be evaluated as $2 a_{\text {mod }}=2\left(\mathrm{v}_{\mathrm{p}} / \omega_{\mathrm{Bh}}\right) \cdot 250 / 370 \approx 6.8 \mathrm{v}_{\mathrm{Ap}}$, which is in reasonable agreement with the simulations. Another mode is generated because protons travel not strictly along the $X$-axis, but also gyrate. This provides a beam propagating along the proton cycloid, and a wave with a speed $\left(\omega / k_{x}\right)$ of about $\mathrm{v}_{\mathrm{ph}, 2_{x}} \approx$ $2 a_{\mathrm{p}} \omega_{\mathrm{Bp}}$, where $2 a_{\mathrm{p}}$ is the height of the proton cycloid, is expected. The height of the proton cycloid is at $m_{\mathrm{p}} n_{\mathrm{p}} / m_{\mathrm{h}} n_{\mathrm{h}} \approx$ 4 times less than that the corresponding height of the trajectory for heavies because of the momentum and energy conservation $\left(\Delta \mathrm{v}_{\mathrm{p}} \approx\left(m_{\mathrm{h}} n_{\mathrm{h}} / m_{\mathrm{p}} n_{\mathrm{p}}\right) \cdot \mathrm{v}_{\mathrm{p}}\right)$. Then, the phase velocity $\mathrm{v}_{\mathrm{ph}, 2_{x}} \approx 6.8 \mathrm{v}_{\mathrm{Ap}} \cdot 0.25 \approx 1.7 \mathrm{v}_{\mathrm{Ap}}$. The phase velocity of the third wave is $\mathrm{v}_{\mathrm{ph}, 3_{x}} \approx 6.8 \mathrm{v}_{\mathrm{Ap}}-1.5 \mathrm{v}_{\mathrm{Ap}}=5.3 \mathrm{v}_{\mathrm{Ap}}$. Both values are in a reasonable agreement with simulations.

It is worth noting that in LLR systems, the Lorenz force related with the relative motion of both ion species becomes important. Moreover, the motion of heavies is mostly affected by the action of the Lorentz force $(1 / c)\left(n_{\mathrm{p}} / n_{\mathrm{e}}\right) \Delta \mathbf{v} \times \boldsymbol{B}$ (the first term on the left-hand side of the momentum equation for heavies). Indeed, the ratio of the Lorentz force term, related with relative motion of ions, $(e / c)\left(n_{\mathrm{p}} / n_{\mathrm{e}}\right)(\Delta \mathrm{v} /$ $\left.m_{\mathrm{h}}\right) B$ to the pressure term $\nabla B^{2} / m_{\mathrm{h}} 8 \pi n_{\mathrm{e}}$ is about of $2(\Delta \mathrm{v} /$ $\left.\mathrm{v}_{\mathrm{Ap}}\right)\left(\omega_{\mathrm{Bp}} / V_{\mathrm{Ap}}\right) l$, where $l$, is a characteristic scale of the system and $L$ is a proton inertial skin length $L=V_{\mathrm{Ap}} / \omega_{\mathrm{Bp}}$. For the case of $\Delta \mathrm{v} \cong \mathrm{v}_{\mathrm{p}}$, the ratio is $2(l / L) M_{\mathrm{A}} \gg>1$. If $\Delta \mathrm{v} \cong \mathrm{v}_{\mathrm{A}}$, the ratio of terms is also large because $l>l$. In the momentum equation for protons, the pressure term may be dropped only when the number density of heavy ions is not very low $\left(2\left(\Delta \mathrm{v} / \mathrm{v}_{\mathrm{Ap}}\right)(l / L)\left(n_{\mathrm{h}} / n_{\mathrm{e}}\right)>>1\right)$. The differential speed $\Delta \mathrm{v}$ may be evaluated from the momentum equations by neglecting of pressure terms:

$$
\frac{d \mathrm{v}_{\mathrm{p}}}{d t}=\omega_{\mathrm{Bp}} \frac{n_{\mathrm{h}}}{n_{\mathrm{e}}}\left(\mathrm{v}_{\mathrm{p}}-\mathrm{v}_{\mathrm{h}}\right) \times b
$$

$$
\frac{d \mathrm{v}_{\mathrm{h}}}{d t}=\omega_{\mathrm{Bh}} \frac{n_{\mathrm{p}}}{n_{\mathrm{e}}}\left(\mathrm{v}_{\mathrm{h}}-\mathrm{v}_{\mathrm{p}}\right) \times b
$$

or

$$
\frac{d \Delta \mathrm{v}}{d t}=\Omega^{*} \Delta \mathrm{v} \times b
$$

where $\Omega^{*}=\omega_{\mathrm{Bp}}\left(n_{\mathrm{h}} / n_{\mathrm{e}}\right)+\omega_{\mathrm{Bh}}\left(n_{\mathrm{p}} / n_{\mathrm{e}}\right)$ is the bi-ion cut-off frequency with which both ion groups gyrate around each other.

Therefore, the elimination of the force related with the differential streaming of ion flows is not well justified. The dispersion relation becomes more complicated, but the mechanism of bunching remains the same. For a "heavy ion beam" in a transverse magnetic field, bunching is mostly provided by the Lorentz force $(e / c)\left(\Delta \mathrm{v}_{\perp} \times B\right)_{x}$ which oscillates with $\Omega^{*}$ frequency. This oscillating motion excites the so-called bi-ion waves and the resonance condition in the proton reference frame has the form:

$$
\omega-k \mathrm{v} \approx-\Omega^{*}
$$

The characteristic wavelength of bunching may be approximately evaluated in the following way. A stabilization of beam instability occurs due to the trapping of particles in the potential troughs of the waves:

$$
\gamma \tau_{\text {trap }} \approx 1
$$

where $\gamma$ is the growth rate of the instability and $\tau_{\text {trap }}$ is the time of the trapping

$$
\tau_{\text {trap }} \approx \frac{1}{k \mathrm{v}_{x}} \approx \frac{1}{k(e \varphi / m)^{1 / 2}} .
$$

Here $\varphi=E / k \approx \Delta \mathrm{v} B / c k$. Then $\gamma \approx\left(k \Delta \mathrm{v} \Omega^{*}\right)^{1 / 2}$. On the other hand, the maximum growth rate occurs at $\Delta \mathrm{v} \approx c_{\mathrm{Mp}}\left(c_{\mathrm{Mh}}<<\right.$ $\left.c_{\mathrm{Mp}}\right)$ and is given by $\gamma / k=c_{\mathrm{MS}} *$, where $c_{\mathrm{MS}} *$ is the magnetosound speed based on the geometrical mean of the proton and heavy ion masses (McKenzie et al., 1993):

$$
c_{\mathrm{MS}}^{*}=\sqrt{c_{\mathrm{Mp}} c_{\mathrm{Mh}}} \cdot
$$

This gives a granulation scale of the heavy ion flow:

$$
\lambda=\frac{2 \pi}{k} \approx \frac{\left(c_{\mathrm{MS}}^{*}\right)^{2}}{c_{\mathrm{Mp}} \Omega^{*}} \approx \frac{2 \pi \mathrm{v}_{\mathrm{p}}}{\Omega^{*}} \frac{\left(c_{\mathrm{MS}}^{*}\right)^{2}}{c_{\mathrm{Mp}} \mathrm{v}_{\mathrm{p}}} \approx \frac{S}{n} .
$$

Here $S=2 \pi \mathrm{v}_{\mathrm{p}} / \Omega *$ is the wavelength of the cycloid, and $n=$ $\mathrm{v}_{\mathrm{p}} c_{\mathrm{Mp}} /\left(c_{\mathrm{MS}} *\right)^{2}=\mathrm{v}_{\mathrm{p}} / c_{\mathrm{Mh}}$ is a number of bunches. For plasma parameters used in the 1-D simulations (the localized obstacle, $\left.m_{\mathrm{h}}=12 m_{\mathrm{p}}, \beta=c_{\mathrm{sp}}{ }^{2} / \mathrm{v}_{\mathrm{Ap}}{ }^{2}=1\right)$, the number of bunches in the cycloid wavelength is about 50 and the step between bunches is about $\lambda \approx 2 \pi / k \sim 8 L$. This value differs by a factor of 2 from the 1-D simulations. 
The characteristic wavelength may be also written in the form:

$$
\lambda \approx \frac{2 \pi \mathrm{v}_{\mathrm{Ap}}}{\omega_{\mathrm{Bp}}}\left(\frac{m_{\mathrm{h}}}{m_{\mathrm{p}}}\right)^{1 / 2}\left(\frac{n_{\mathrm{h}}}{n_{\mathrm{p}}}\right)^{1 / 2}=2 \pi L\left(\frac{m_{\mathrm{h}}}{m_{\mathrm{p}}}\right)^{1 / 2}\left(\frac{n_{\mathrm{h}}}{n_{\mathrm{p}}}\right)^{1 / 2}
$$

i.e., the wavelength increases with the mass of heavies as $\sqrt{m_{\mathrm{h}} / m_{\mathrm{p}}}$. This dependence was confirmed by simulations performed with different masses of heavy ions (Sauer et al., 1996a).

The frequency of oscillations in the heavies in the reference frame of the proton flow is about $\omega \approx k \Delta v-\omega_{\text {beam }}$, where $\omega_{\text {beam }} \approx \Omega^{*}$. Near the nodes of the cycloid, where the heavies are near the rest, $\Delta \mathrm{v}=-\mathrm{v}_{\mathrm{p}}$ and the frequency of the waves in the laboratory frame is $\omega_{\mathrm{Lab}} \approx \omega+k \mathrm{v}_{\mathrm{p}} \approx \Omega^{*}$. In the general case, the frequency of bi-ion bunches is mainly determined by the Doppler term $\omega \sim k \mathrm{v}_{\mathrm{h}}$.

The 1-D hybrid simulations show that compressional magnetosound waves gradually steepen and give rise to shocklets. The concept of multiple shocks at comets or nonmagnetized planets was first discussed by Omidi and Winske (1990). It was suggested that the transition region of the comet Giacobini-Zinner consists of a series of steepened waves, each of which resulted in the heating and deceleration of the solar wind plasma until it became subsonic in the comet's reference frame. The presented results show that the multiple shocks arising from different wave modes are split due to a difference in their speed in the laboratory reference system. Proton shocklets are generated by steepened proton magnetosound waves. Heavy ion shocks take their origin from the slow modes which are carried by an ion beam and resonantly interact with proton waves. 2-D bi-ion MHD simulations (Sauer et al., 1998) allowed to trace these waves for the case of the solar wind interaction with Mars. "Proton shocks" give rise to a bow shock, and "heavy ion shocks" form additional MHD-discontinuities in the magnetosheath of Mars (Sauer et al., 1996b).

\section{Conclusions}

One-dimensional hybrid simulations show that a differential motion of ion species leads to the effect of bunching. A bunching of plasma arises due to a resonant interaction between waves, generated in the "proton plasma" by a heavy ion beam, and slow waves in a beam. Slow waves, which are carried by a beam and interact with plasma waves, are bi-ion waves driven by the Lorentz and pressure forces. They are waves of a negative energy and grow due to the energy transfer to the waves excited in "proton plasma". A characteristic step between bunches is about 20 proton inertial lengths. Both types of waves steepen and gradually evolve to shocklets. Two types of shocks were identified. One of them, a "proton shock" has a bow shock as the counterpart in the 2-D case. "Heavy ion shocks" form the heavy ion discontinuities (HIDs).

Acknowledgments. The authors wish to express their thanks to the ISSI in Bern for the support and collaboration within the framework of the Visiting Science Program. A comprehensive multi-instrument study of Martian plasma environment and the comparison with a modeling initiated in the framework of the Visiting Science Program of the International Space Science University (ISSI) in Bern (Switzerland) gave the opportunity to consider the problem of bi-ion interaction from observational and simulation points of view.

\section{References}

Bogdanov, A., K. Sauer, K. Baumgärtel, and K. Srivastava, Plasma structures at weak outgassing comets. Results from bi-ion fluid analysis, Planet. Space Sci., 44, 519-528, 1996.

Brinca, A. L. and B. T. Tsurutani, Oblique behavior of low frequency electromagnetic waves excited by newborn cometary ions, J. Geophys. Res., 94, 3-14, 1989

Dubinin, E., K. Sauer, R. Lundin, K. Baumgärtel, and A. Bogdanov, Structuring of the transition region (plasma mantle) of the Martian magnetosphere, Geophys. Res. Lett., 23, 785-788, 1996.

Galeev, A. A., R. Z. Sagdeev, V. D. Shapiro, V. I. Shevchenko, and K. Szego, Quasilinear theory of the ion cyclotron instability and its application to the cometary plasma, in Cometary Plasma Processes, edited by A. D. Johnstone, pp. 223-240, Geophysical Monograph 61, 1991.

Gary, S. P. and C. D. Madland, Electromagnetic ion instabilities in a cometary environment, J. Geophys. Res., 93, 235-241, 1988.

McKenzie, J. F., E. Marsch, K. Baumgärtel, and K. Sauer, Wave and stability properties of multi-ion plasmas with applications to winds and flows, Ann. Geophys., 11, 341-353, 1993.

Omidi, N. and D. Winske, A kinetic study of solar wind mass-loading and cometary bow shocks, J. Geophys. Res., 92, 13409-13426, 1987.

Omidi, N. and D. Winske, Steepening of kinetic magnetosonic waves into shocklets: simulations and consequences for planetary shocks and comets, J. Geophys. Res., 95, 2281-2300, 1990.

Omidi, N., H. Karimabadi, D. Krauss-Varban, and K. Killen, Generation and nonlinear evolution of oblique magnetosonic waves: application to foreshock and comets, in Solar System Plasmas in Space and Time, edited by J. L. Burch and J. H. Waite, Jr., pp. 71-84, Geophysical Monograph 84, 1994

Sauer, K., A. Bogdanov, K. Baumgärtel, and E. Dubinin, Bi-ion discontinuities at weak solar wind mass-loading, Physica Scripta, T63, 111-118, 1996a.

Sauer, K., E. Dubinin, and K. Baumgärtel, Bow shock splitting in bi-ion flows, Geophys. Res. Lett., 23, 3643-3646, 1996 b.

Sauer, K., E. Dubinin, and K. Baumgärtel, Bi-ion structuring in the magnetosheath of Mars. Theoretical modelling, Adv. Space Res., 20, (2)137-(2)142, 1997.

Sauer, K., E. Dubinin, and K. Baumgärtel, Nonlinear MHD-waves and discontinuities in the Martian magnetosheath. Observations and 2D biion simulations, Earth Planets Space, 1998 (submitted).

E. Dubinin (e-mail: dubinin@helene.mpae.gwdg.de), K. Sauer (e-mail: sauer@linax1.mpae.gwdg.de), K. Baumgärtel (e-mail: kbaumgaertel@aip.de), and K. Srivastava 